This item was submitted to Loughborough's Research Repository by the author.

Items in Figshare are protected by copyright, with all rights reserved, unless otherwise indicated.

\title{
Biomechanical analysis of ankle ligamentous sprain injury cases from televised basketball games: understanding when, how and why ligament failure occurs
}

\section{PLEASE CITE THE PUBLISHED VERSION}

http://dx.doi.org/10.1016/j.jsams.2017.05.006

PUBLISHER

(C) 2017 Sports Medicine Australia. Published by Elsevier Ltd

\section{VERSION}

AM (Accepted Manuscript)

\section{PUBLISHER STATEMENT}

This work is made available according to the conditions of the Creative Commons Attribution-NonCommercialNoDerivatives 4.0 International (CC BY-NC-ND 4.0) licence. Full details of this licence are available at: https://creativecommons.org/licenses/by-nc-nd/4.0/

\section{LICENCE}

CC BY-NC-ND 4.0

\section{REPOSITORY RECORD}

Panagiotakis, Emmanouil, Kam-Ming Mok, Daniel Fong, and Anthony M.J. Bull. 2017. "Biomechanical Analysis of Ankle Ligamentous Sprain Injury Cases from Televised Basketball Games: Understanding When, How and Why Ligament Failure Occurs". Loughborough University. https://hdl.handle.net/2134/25252. 
Title:

Biomechanical analysis of ankle ligamentous sprain injury cases from televised basketball games: understanding when, how and why ligament failure occurs Authors:

Emmanouil Panagiotakis ${ }^{a}$, Kam-Ming Mok $^{b, c}$, Daniel Tik-Pui Fong ${ }^{\mathrm{d}}$, Anthony M.J. Bull ${ }^{\mathrm{a}}$

Affiliations:

${ }^{\mathrm{a}}$ Department of Bioengineering, Imperial College, London, United Kingdom

${ }^{b}$ Department of Sports Medicine, Oslo Sports Trauma Research Center, Norwegian school of Sports Medicine, Oslo, Norway

${ }^{\mathrm{C}}$ Department of Orthopaedics and Traumatology, Faculty of Medicine, The Chinese University of Hong Kong, Hong Kong SAR

${ }^{d}$ National Centre for Sport and Exercise Medicine - East Midlands, School of Sport, Exercise and Health Sciences, Loughborough University, Leicestershire, United Kingdom

Corresponding Author:

Anthony Bull, a.bull@imperial.ac.uk 
1 Title:

2

3

4

5

Biomechanical analysis of ankle ligamentous sprain injury cases from televised basketball games: understanding when, how and why ligament failure occurs 


\section{Abstract}

7 Objectives. Ankle sprains due to landing on an opponent's foot are common in basketball.

8 There is no analysis to date that provides a quantification of this injury mechanism. The aim

9 of this study was to quantify the kinematics of this specific injury mechanism and relate this

10 to lateral ankle ligament biomechanics.

Design. Case series.

Methods. The Model-Based Image-Matching technique was used to quantify calcaneofibular-talar kinematics during four ankle inversion sprain injury incidents in televised NBA basketball games. The four incidents follow the same injury pattern in which the players of interest step onto an opponent's foot with significant inversion and a diagnosed ankle injury. A geometric analysis was performed to calculate the in vivo ligament strains and strain rates for the anterior talofibular ligament (ATFL) and the calcaneofibular ligament (CFL).

Results. Despite the controlled selection of cases, the results show that there are two distinct injury mechanisms: sudden inversion and internal rotation with low levels of plantarflexion; and a similar mechanism without internal rotation. The first of these mechanisms results in high ATFL and CFL strains, whereas the second of these strains the CFL in isolation.

Conclusions. The injury mechanism combined with measures of the ligament injury in terms of percentage of strain to failure correlate directly with the severity of the injury quantified by return-to-sport. The opportunity to control excessive internal rotation through proprioceptive training and/or prophylactic footwear or bracing could be utilised to reduce the severity of common ankle injuries in basketball.

Keywords: injury mechanism; ankle; return-to-sport; inversion; internal rotation 
31 The ankle is the most widely injured part of the body during sport, accounting for $10 \%$ to $30 \%$ of all sport-related injuries ${ }^{1,2,3,4}$ and ankle injuries sustained by athletes create an annual healthcare burden of over $\$ 4$ billion in the U.S alone. ${ }^{5}$ The most common ankle injuries involve the lateral ligaments. ${ }^{2}$. Lateral ligament injuries in basketball players can cause significant reduction in playing ability ${ }^{6}$. that may result in match defeats and economic loss to the individual and the team. Understanding the injury mechanism in detail would allow the development of new preventative strategies and the design of protective equipment for basketball players. ${ }^{7}$

The Model-based image-matching (MBIM) technique utilises uncalibrated video sequences to reconstruct three-dimensional human motion patterns and estimate temporal joint angle histories, velocities and accelerations. ${ }^{8}$. This method has been applied in two different studies, which aimed to explore the biomechanics of five actual ankle injuries from televised tennis competitions ${ }^{7}$ and another two injuries during the 2008 Beijing Olympics. ${ }^{9}$ Both studies reported the peak values of ankle joint internal rotation and inversion, such as the values of inversion velocity. The results indicated that ankle ligament injuries resulted from the combination of internal rotation and sudden inversion of the ankle joint, while

47 plantarflexion was absent. ${ }^{7,9,10}$. While kinematics are very important for understanding the injury mechanism of an injury, there has been no similar analysis to date that quantifies ligament loading patterns during injury in a quantitative manner, including, for example, ligament strain or strain rate.

51 Therefore, the aim of this study was to quantify the detailed injury mechanism of the ankle during real ankle injury cases by quantifying ankle kinematics, in vivo ligament strains, strain rates and loading. 
A single common injury mechanism was selected in which a large unwanted ankle inversion secondary to inadvertently stepping onto an opponent's foot during an elite level basketball game was experienced. The inclusion criteria were: conforming to the selected injury mechanism; the player was unable to continue playing after the injury or had problems playing (following the approach taken by Fong and Wei ${ }^{10}$ ); the injury was reported as an ankle sprain injury in the post-match report; two camera views of the incident were available (showing the shank and foot segment clearly and showing an extreme inversion sparin motion) with a video resolution of at least $640 \times 360$ pixels with a frame rate of at least $25 \mathrm{~Hz}$ (the minimum frame rate deemed appropriate in prior work ${ }^{7}$ ), and the basketball game was of an elite level. Four cases that occurred during televised NBA basketball games were available.

In order to present and compare the results for four different cases, which have different time lengths, time-normalisation was employed. The start point was defined as the time (frame) of first contact between the player of interest's injured foot and the opponent's foot. The end point was defined as the time (frame) when the player of interest's injured foot does not have any contact with the opponent's foot or the ground. The dependent variables were then normalised to the percentage of the injury incident.

The videos were trimmed and edited in order to create uncompressed $\mathrm{AVI}$ image sequences for each camera view with Adobe Premiere Pro software (version CS5.5, Adobe Systems Inc., San Jose, CA, USA ). Then, AVI image sequences were merged and rendered into a synchronised video sequence by Adobe After Affects (version CS5.5, Adobe Systems Inc., San Jose, CA, USA).

An anthropometric data figure was used in order to calculate the lengths of each lower limb segment (foot length and breadth, shin length and thigh length) relative to the total height of each basketball player (source: www.nba.com) and build a skeleton model for the matching 
process. The skeleton model from Zygote Media Group Inc. was used. The skeletal structures and court dimensions were matched to the video images using Poser 4 and Poser Pro Pack (Curious Labs, Inc., Santa Cruz, CA, USA) software. The dimensions of the basketball court in each case were obtained from the National Basketball Association to construct a virtual environment.

The virtual environment was manually matched to the image background for each frame in every camera view, using a key frame and spline interpolation technique by adjusting the camera calibration parameters: position, orientation and focal length. The skeleton model used for the skeleton matching of the lower limb consisted of four rigid segments: foot, tibia/fibula, thigh and pelvis. The complete matching process is fully described by Krosshaug and Bahr. ${ }^{8}$ The adjustment of Mok et al. ${ }^{11}$. was used to define the ankle joint centre, following the International Society of Biomechanics (ISB) recommendation. ${ }^{12}$. Finally, frame by frame adjustments were made to ensure smooth motion of the cameras and the skeleton for each case (Figure 1).

Ankle joint kinematics data were calculated from the skeletal matching data. Poser 4 and Poser Pro Pack were used to export the ankle joint angle histories that were subsequently imported into a custom-written Matlab scripts to compute joint angles according to a Joint Coordinate System method ${ }^{13}$, following the ISB recommendations ${ }^{12}$.

Data were filtered and interpolated by Woltring's generalised cross-validation spline package with $15 \mathrm{~Hz}$ cut-off frequency. The kinematic data were then used to quantify lateral ankle ligament length changes to then calculate the ligament strains and strain rates to infer injury data. The two key ligaments that are loaded during the proscribed injury mechanism were identified, these are the anterior talofibular ligament (ATFL) and the calcaneofibular ligament (CFL). Within the virtual environment in Poser 4, the anatomical insertion points of the two ligaments were identified and marked with spherical features (Figure 2). The insertion 
positions were located for each time frame. The unloaded lengths of the ligaments $\left(L_{0}\right)$ were calculated with the skeleton orientated in the standing position and then ligament lengths were calculated as the linear distance between insertion points for each time step, following the 'minimal recruitment length' approach of Blankevoort et al. ${ }^{14}$

$<$ FIGURE 2>

Engineering strain at each point was calculated as the ratio of length change over original length

Engineering strain rate was calculated as follows:

Maximum Load, stiffness, deflection to failure and strain to failure across the strain rates experienced were calculated for both ligaments using scaled data from the literature. Attarian et $\mathrm{al}^{15}$. found that the ATFL and CFL had stiffnesses of $272 \pm 46 \mathrm{~N} / \mathrm{cm}$ and $549 \pm 88$ $\mathrm{N} / \mathrm{cm}$, respectively from a mean donor age of 57.9 years, loaded at strain rates of $96 / \mathrm{s}$ and 61/s. Recent work has shown that there is no strain rate effect on maximum stress and ultimate load for ligaments when loaded above $1 / \mathrm{s}^{16}$ and the expected strain rates in the four cases are above $1 / \mathrm{s}$, therefore no strain rate scaling is required for the above data.

The ultimate load of ligaments decreases with age according to an exponential decay ${ }^{17}$ : . This was use to scale the properties from Attarian et al ${ }^{15}$ to the mean age of the four cases in this study (29 years), with $\lambda=0.2$ and $t=29$.

Woo et $\mathrm{al}^{17}$. found that stiffness decreased by $16 \%$ from a young age group to an older age group, therefore the stiffness data, deflection to failure and strain to failure of the ATFL and the CFL from the Attarian et $\mathrm{al}^{15}$. data were scaled for the younger group (Table 1). 
All four injuries are described below.

131 Case 1: The injured player suffered from a left ankle sprain in a game for the NBA's regular season. His team announced that he had suffered from a sprain and a bone bruise and was ruled out for two weeks.

Case 2: The injured player suffered from a right ankle injury in the first half in Game 3 of the Eastern Conference NBA Quarterfinals. He returned later on in that game in obvious discomfort and played the following three games on playing time restriction with a significantly reduced performance. These were the last games in the season and further information on the injured player's rehabilitation was not available.

Case 3: The injured player sprained his right ankle in the first quarter in this year's Game 4 of the Western Conference NBA Finals. He tried to play on, however, in obvious pain, missed the rest of the game. He played the following game without time restriction.

Case 4: The injured player sprained his left ankle in Game 3 of the Eastern Conference NBA Quarterfinals. He was ruled out of playing for at least 3 months. Due to usual restrictions on medical data from elite athletes, no medical imaging and orthopaedic reports were available.

Results

Case data and all quantitative results are presented in Table 2.

Ankle kinematics and ligament strains are presented in Figure 3, demonstrating that all cases exhibit a high level of inversion $\left(>70^{\circ}\right)$ with no plantarflexion. Case 1 has very little internal rotation and all other cases exhibited large internal rotation $\left(>25^{\circ}\right)$. All cases

151 demonstrate similar maximum strains for the CFL, with Case 1 having low strains for the 152 ATFL when compared to the other three cases. 
154 The case analysis below proposes the injury magnitude through an analysis of maximum strain to the ATFL and CFL. This is recorded as "injury assessment" in Table 2.

Case 1: The maximum ATFL strain was $18 \%$, which is significantly below the strain to failure. The maximum CFL strain was $61 \%$, which exceeds the strain to failure, suggesting a complete rupture of the CFL. The player was ruled out of sport for two weeks.

Case 2: The maximum ATFL strain was $71 \%$, which is approximate at the strain to failure (67\%). The maximum CFL strain was $47 \%$, which is equal to the approximate strain to failure. The most probably outcome, therefore, was that the CFL and ATFL each sustained minor sprains. The player continued to play below his normal standard.

Case 3: The maximum ATFL strain was $47 \%$, which is below the strain to failure. The maximum CFL strain was 53\%, above the strain to failure. The most likely outcome was that the ATFL was kept intact and the CFL was moderately sprained. The player returned to action after only two days.

Case 4: The maximum ATFL strain value was $73 \%$, which is just greater than the strain to failure (67\%). The maximum CFL strain was $49 \%$, also slightly over the strain to failure (47\%), suggesting that both ligaments sustained moderate sprains, similar to Case 2 , at a slightly more severe level. The player was ruled out of sport for three months.

\section{Discussion}

All cases analysed here follow a similar pattern. The main factor causing the injury is that the injured player steps onto an opponent's foot (abnormal landing) to create an ankle injury. The consistent features in these injury patterns are a sudden inversion and low values (10$35^{\circ}$ ) of plantarflexion. The lack of plantarflexion indicates that the subtalar joint had little involvement in the injury mechanism. There was great variability in peak internal rotations (3- 
$17747^{\circ}$ ) across the four cases. These results are similar to those in the literature ${ }^{7,9,18}$, however, 178 very low internal rotation has not been shown previously.

179 Basketball is a sport that requires frequent jumps and landings, cutting manoeuvres and 180 contact with other players and thus observing different injury mechanisms is expected. 181 However, the short injury duration and high inversion velocities in all cases indicate that the preventative measures should focus on resisting the inversion torque at the ankle joint for a very short period of time. Proposed mechanisms to achieve this include neuromuscular training on correct foot landing ${ }^{19}$, shoe design such as higher ankle support, and myoelectric anti-sprain stimulation ${ }^{20}$.

This study has a number of key limitations, in particular, we were limited by the number of cases. The minimum frame rate in this study was $25 \mathrm{~Hz}^{7}$, and, although this has been previously deemed appropriate for such analyses, a higher frame rate and higher resolution of the images would also greater resolution and accuracy for the measures of ankle kinematics and ligament strains. The manual skeletal scaling and matching process is subject to user experience and this was conducted by the most experienced member of the team. In addition, the estimation of the rehabilitation time period of each player was based on injury reports from online sources and detailed medical information was not available. The geometric analysis presumes a straight line between ligament insertions without accounting for any possible wrapping. In these cases this wrapping is expected to be negligible.

\section{Conclusion}

A quantitative analysis was performed to identify and calculate ankle joint kinematics and ligament strains in a specific injury mechanism in elite level basketball. We noted two distinct injury mechanismsin our case series: sudden inversion and internal rotation with low levels of plantarflexion; and a similar mechanism without internal rotation. The hypothesise that the first of these mechanisms results in ATFL and CFL sprains or ruptures, whereas the second 
of these damages the CFL in isolation. A link between return-to-sport and ligament strain

204 parameters may be inferred from this work, but this cannot be proven without an appropriate 205 medical history.

206 Practical Implications

207

208

209

210

211

212

213

214 Acknowledgments

215

No financial support was provided for this study. ligament and the anterior talofibular ligament faster return to sport.

- The specific injury mechanism of landing on an opponent's foot can produce isolated rupture of the calcaneofibular ligament of the ankle or a combined rupture of this

- This injury mechanism consists of excessive internal rotation and inversion.

- Reducing internal rotation alone through proprioceptive training and/or prophylactic footwear or bracing will protect the anterior talofibular ligament thus facilitating a 
1. Fong D.T.P., Man C.Y., Yung P.S.H., et al., 2008. Sport-Related Ankle Injuries Attending an Accident Emergency Department. Injury 39, pp. 1222-1227

2. Garrick J.G., 1977. The Frequency of Injury, Mechanism of Injury, and Epidemiology of Ankle Sprain. Am J Sports Med 5, pp. 241-242.

3. Garrick J.G. and Requa R.K. 1988. The Epidemiology of Foot and Ankle Injuries in Sport. Clin Sports Med 7, pp. 29-36.

4. Payne K.A., Berg K., Latin R.W., 1997. Ankle Injuries and Ankle Strength, Flexibility and Proprioception in College Basketball Players. J Athl Train 32, pp. 221-225.

5. Waterman B.R., Owens B.D., Davey S., et al., 2010. The Epidemiology of Ankle Sprains in the United States. J Bone Joint Surg Am 92, pp. 2279-2284.

6. Leanderson J., Nemeth G., Eriksson E., 1993a. Ankle Injuries in Basketball Players. Knee Surg Sports Traumatol Arthrosc 1, pp. 203-205.

7. Fong D.T.P., Ha S.C.W., Mok K.M., et al., 2012. Kinematics Analysis of ankle Inversion Ligamentous Sprain Injuries in Sports: Five Cases from Televised Tennis Competitions. Am J Sports Med 40, pp.2627-2632.

8. Krosshaug T. and Bahr R., 2005. A Model-Based Image-Matching Technique for Threedimensional Reconstruction of Human Motion from Uncalibrated Video Sequences. $J$ Biomech 38, pp. 919-929.

9. Mok K.M., Fong D.T.P., Krosshaug T., et al., 2011b. Kinematics Analysis of ankle Inversion Ligamentous Sprain Injuries in Sports: 2 Cases During the 2008 Beijing Olympics. Am J Sports Med 39, pp. 1548-1552. 
10. Fong D.T.P. and Wei F., 2012. The Use of Model Matching Video Analysis and Computational Simulation to Study the Ankle Sprain Injury Mechanism. International J Adv Robotic Systems 9, pp. 97.

11. Mok K.M., Fong D.T.P., Krosshaug T., et al., 2011a. An Ankle Joint Model-Based Image-Matching Motion Analysis Technique. Gait Posture 34, pp. 71-75.

12. Wu G., Siegler S., Allard P., et al., 2002. ISB Recommendation on Definitions of Joint Coordinate System of Various Joints for the Reporting of Human Joint Motion- part 1: Ankle, Hip and Spine. J Biomech 35, pp. 543-548.

13. Grood E.S. and Suntay W.J., 1983. A Joint Coordinate System for the Clinical Description of Three-Dimensional Motions: Application to the Knee. J Biomech Eng 105, pp. 136-144.

14. Blankevoort L, Huiskes R, de Lange A., 1991 Recruitment of knee joint ligaments. J Biomech Eng 113, pp. 94-103.

15. Attarian D.A., McCrackin H.J., DeVito D.P., et al., 1985. Biomechanical Characteristics of Human Ankle Ligaments. Foot Ankle Intl 6, pp. 54-58.

16. Bonner T.J., Newell N., Karunaratne A., et al., 2014. Strain-Rate Sensitivity of the Lateral Collateral Ligament of the Knee. J Mech Behav Biomed Mater 41, pp 261-270.

17. Woo S.L., Hollis J.M., Adams D.J., et al., 1991. Tensile Properties of the Human FemurAnterior Cruciate Ligament-Tibia Complex: The Effects of Specimen Age and Orientation. Am J Sports Med 19, pp. 217-225.

18. Colville M.R., Marder R.A., Boyle J.J. et al., 1990. Strain Measurement in Lateral Ankle Ligaments. Am J Sports Med 18, pp. 196-200.

19. Verhagen E., van der Beek A., Twisk J., et al., 2004. The effect of a proprioceptive balance board training program for the prevention of ankle sprains: a prospective controlled trial. Am J Sports Med. 32, pp 1385-1393. 
20. Fong D.T., Chu V.W. and Chan K.M., 2012. Myoelectric stimulation on peroneal muscles resists simulated ankle sprain motion. J Biomech. 45, pp. 2055-2057. 


\section{Table Legends}

Table 1: Derived biomechanical data of the anterial talofibular ligament (ATFL) and the calcaneofibular ligament (CFL) for four basketball players with mean age of 29 years old. Table 2: Case data and quantitative results for the 4 different injury cases. 
Table 1

\begin{tabular}{|c|c|c|}
\hline & ATFL & CFL \\
\hline Maximum Load (N) & $245 \pm 40$ & $610 \pm 97$ \\
\hline Stiffness (N/cm) & $368 \pm 62$ & $742 \pm 118$ \\
\hline Deflection to Failure (cm) & 0.67 & 0.82 \\
\hline Strain to Failure (\%) & 64 & 47 \\
\hline
\end{tabular}


Table 2

\begin{tabular}{|c|c|c|c|c|}
\hline & Case 1 & Case 2 & Case 3 & Case 4 \\
\hline Minimum video resolution (pixels) & $1280 \times 720$ & $640 \times 360$ & $1280 \times 720$ & $640 \times 360$ \\
\hline Frame rate $(\mathrm{Hz})$ & 30 & 30 & 30 & 29.9 \\
\hline Player height (m) & 2.11 & 2.03 & 1.91 & 2.11 \\
\hline Player mass (kg) & 111.1 & 102.1 & 94.3 & 105.2 \\
\hline Player age (years) & 33 & 32 & 24 & 27 \\
\hline Injury severity (days of absence) & 14 & $\begin{array}{c}0 \text { (reduced } \\
\text { performance) }\end{array}$ & 2 & 90 \\
\hline Peak Inversion $\left({ }^{\circ}\right)$ & 92.7 & 77.4 & 96.6 & 107.5 \\
\hline Time to Peak Inversion (sec) & 0.17 & 0.13 & 0.23 & 0.23 \\
\hline Peak Internal Rotation $\left(^{\circ}\right)$ & 3.4 & 38.2 & 28.0 & 46.6 \\
\hline Time to Peak Internal Rotation (sec) & 0.1 & 0.13 & 0.17 & 0.4 \\
\hline Peak Plantarflexion $\left(^{\circ}\right)$ & 3.2 & 26.4 & 12 & 53.9 \\
\hline Time to Peak Plantarflexion (sec) & 0.13 & 0.1 & 0.2 & 0.47 \\
\hline Maximum ATFL strain (\%) & 17.6 & 70.9 & 47.1 & 72.5 \\
\hline Time to maximum ATFL strain (sec) & 0.13 & 0.13 & 0.23 & 0.3 \\
\hline Maximum CFL strain (\%) & 60.8 & 46.5 & 53.0 & 48.7 \\
\hline Time to maximum CFL strain (sec) & 0.17 & 0.13 & 0.23 & 0.23 \\
\hline Mean Value of ATFL Strain Rate (/s) & 1.3 & 3 & 1.8 & 2 \\
\hline Mean Value of CFL Strain Rate (/s) & 2.6 & 2.4 & 2 & 1.6 \\
\hline ATFL injury assessment & No & Minor Injury & No & $\begin{array}{l}\text { Moderate } \\
\text { Injury }\end{array}$ \\
\hline CFL injury assessment & $\begin{array}{c}\text { Complete } \\
\text { Rupture }\end{array}$ & Minor Injury & $\begin{array}{l}\text { Moderate } \\
\text { Injury }\end{array}$ & $\begin{array}{l}\text { Moderate } \\
\text { Injury }\end{array}$ \\
\hline
\end{tabular}




\section{Figure Legends}

Figure 1: Virtual environment (basketball court lines and basket were created manually) and skeleton matching (Case 4).

Figure 2: Matching procedure for calculating the lengths of the two key ligaments (Case 1).

Figure 3: Ankle kinematics and ligament strain for four cases of ankle inversion injury 
Figure 1

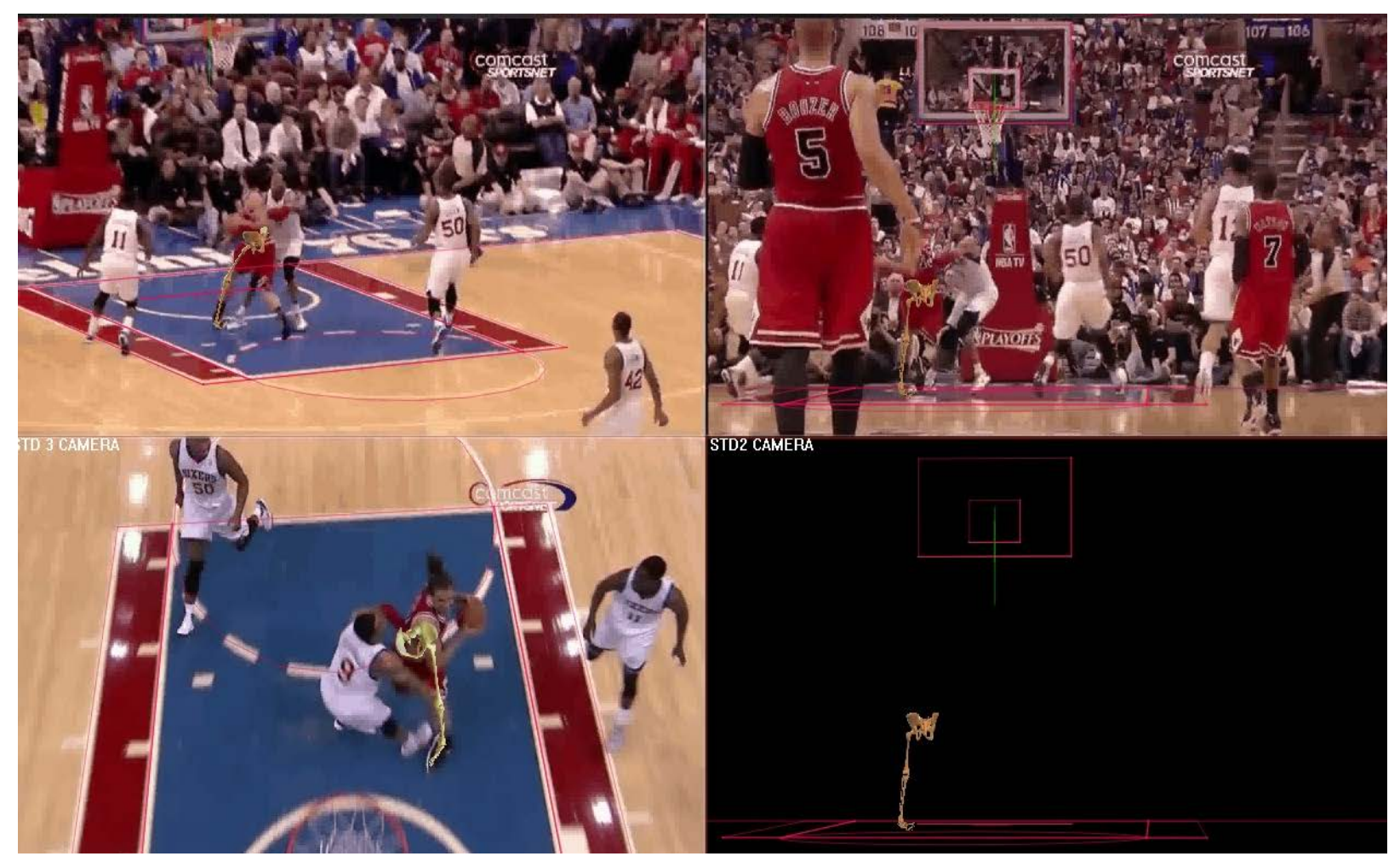


Figure 2

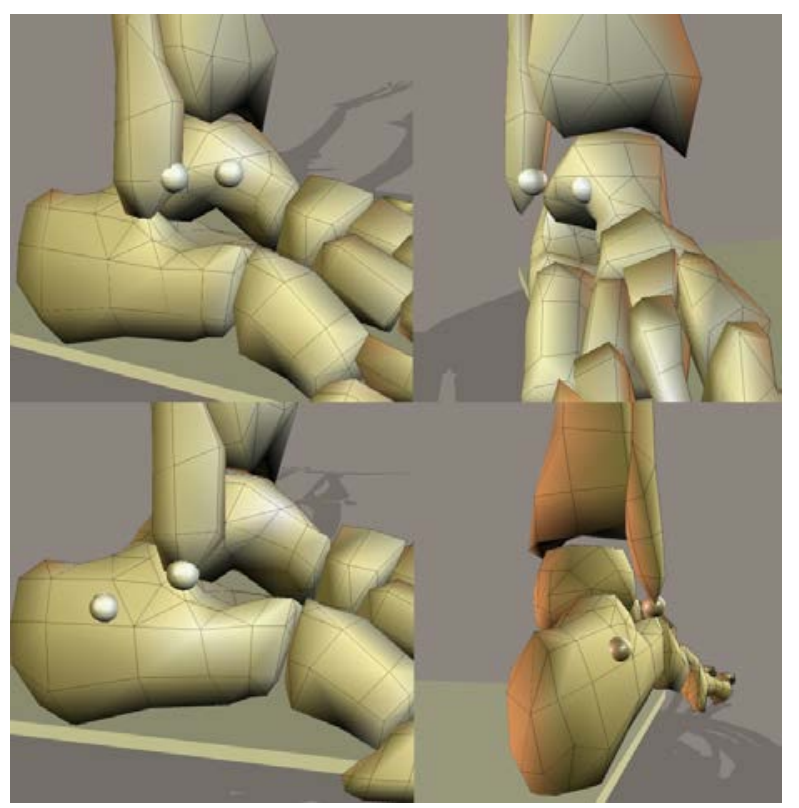


Figure 3
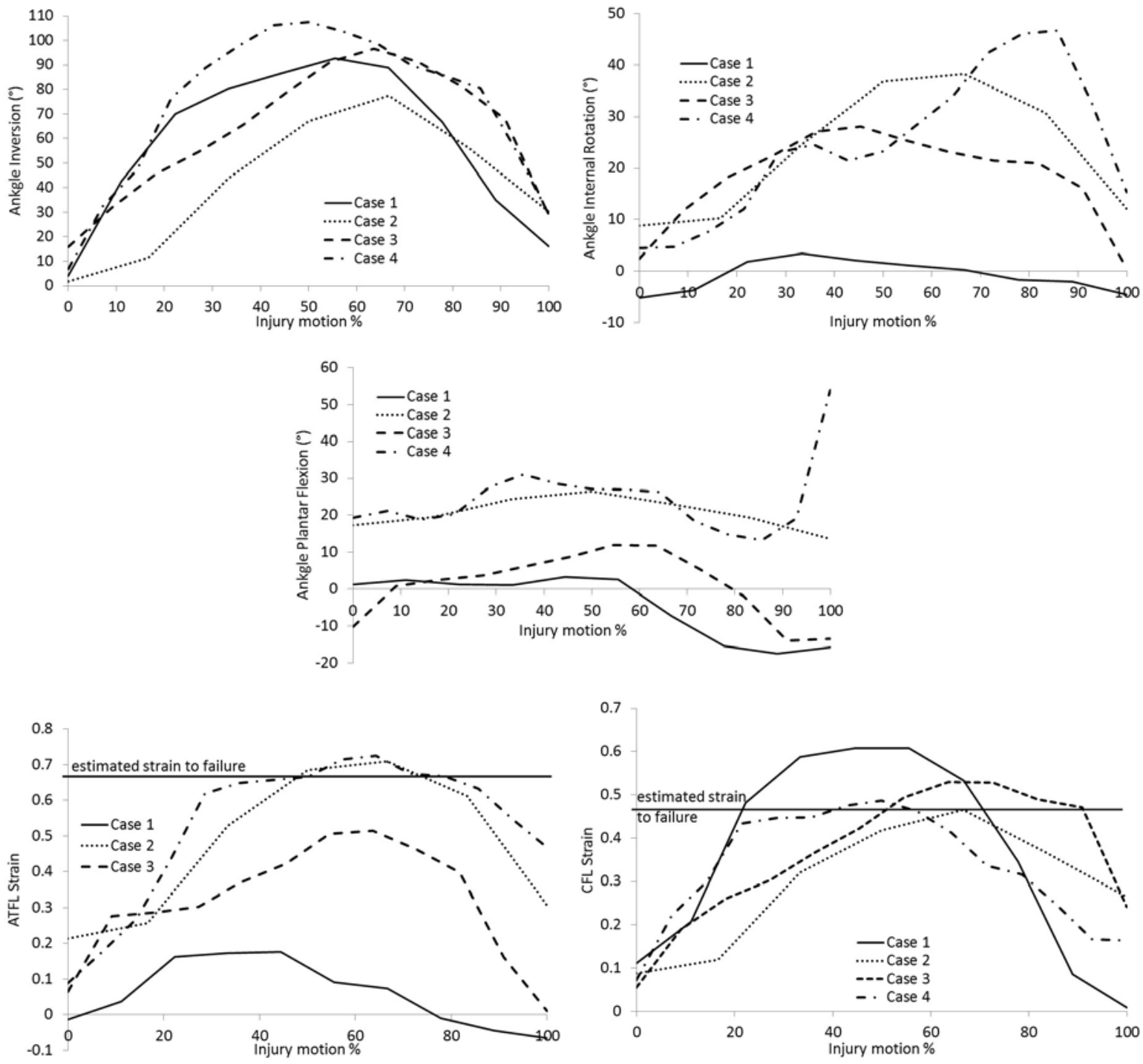